\title{
Global Stability of an Eco-Epidemiological Model with Time Delay and Saturation Incidence
}

\author{
Shuxue Mao, Rui Xu, Zhe Li, and Yunfei Li \\ Institute of Applied Mathematics, Shijiazhuang Mechanical Engineering College, \\ Shijiazhuang 050003, China \\ Correspondence should be addressed to Shuxue Mao, maoshuxue8759@126.com
}

Received 30 June 2011; Accepted 23 August 2011

Academic Editor: Her-Terng Yau

Copyright ( $) 2011$ Shuxue Mao et al. This is an open access article distributed under the Creative Commons Attribution License, which permits unrestricted use, distribution, and reproduction in any medium, provided the original work is properly cited.

\begin{abstract}
We investigate a delayed eco-epidemiological model with disease in predator and saturation incidence. First, by comparison arguments, the permanence of the model is discussed. Then, we study the local stability of each equilibrium of the model by analyzing the corresponding characteristic equations and find that Hopf bifurcation occurs when the delay $\tau$ passes through a sequence of critical values. Next, by means of an iteration technique, sufficient conditions are derived for the global stability of the disease-free planar equilibrium and the positive equilibrium. Numerical examples are carried out to illustrate the analytical results.
\end{abstract}

\section{Introduction}

Recently, more attention has been paid to the eco-epidemiology model which considers both the ecological and epidemiological issues simultaneously due to the fact that most of the ecological populations suffer from various infectious diseases which have a significant role in regulating population sizes (see, e.g., [1-6]). Mukherjee [7] discussed a predator-prey model with disease in prey. The criteria were derived for both local stability and instability involving system parameters. In addition, considering the time required by the susceptible individuals to become infective after their interaction with the infectious individuals, Zhou et al. [8] formulated a delayed eco-epidemiology model and found that the Hopf bifurcation occurs when the delay passes through a sequence of critical values. They also gave an estimation of the length of the time delay to preserve stability. On the other hand, in the predator-prey system, the disease not only can spread in prey but also can spread in predator. Therefore, Zhang et al. [9] studied an eco-epidemiological model with disease in predator and showed that a Hopf bifurcation can occur as the delay increased. The above-mentioned works all used bilinear incidence to model disease transmission. 
Note that ecologically the assumption of standard incidence instead of the former bilinear mass action incidence is meaningful for large populations and a low number of infected individuals, a very good justification behind this assumption being found in [10]. Han et al. [11] proposed four modifications of a predator-prey model with standard incidence to include an SIS or SIR parasitic infection. Thresholds were identified, and global stability results were proved. When the disease persists in the prey population and the predators have a sufficient feeding efficiency to survive, the disease also persists in the predator population. Hethcote et al. [12] considered a predator-prey model including an SIS parasitic infection in the prey with infected prey being more vulnerable to predation. Thresholds were identified which determine when the predator population survives and when the disease remains endemic.

However, there are a variety of factors that emphasize the need for a modification of the bilinear incidence and standard incidence. For example, the underlying assumption of homogeneous mixing may not always hold. Incidence rates that increase more gradually than linearly in $I$ and $S$ may arise from saturation effects. It has been strongly suggested by several authors that the disease transmission process may follow saturation incidence. After studying the cholera epidemic spread in Bari in 1973, Capasso and Serio [13] introduced a saturated incidence rate $g(I) S$ into epidemic models with $g(I)=\beta I /(1+\alpha I)$. A general saturation incidence rate $g(I) S=\beta I^{p} S /\left(1+\alpha I^{p}\right)$ was proposed by Liu et al. [14] and used by a number of authors; see, for example, Ruan and Wang [15] $(p=2)$, Bhattacharyya and Mukhopadhyay [16] $(p=1)$, and so forth. $\beta I^{p}$ measures the infection force of the disease, and $1 /\left(1+\alpha I^{p}\right)$ measures the inhibition effect from the behavioral change of the susceptible individuals when their number increases or from the crowding effect of the infective individuals. This incidence rate seems more reasonable than the bilinear incidence rate $\beta S I$, because it includes the behavioral change and crowding effect of the infective individuals and prevents the unboundedness of the contact rate by choosing suitable parameters.

Motivated by the works of Zhang et al. [9] and Capasso and Serio [13], in this paper, we are concerned with the effect of disease in predator and saturated incidence on the dynamics of eco-epidemiological model. To this end, we consider the following delay differential equations:

$$
\begin{gathered}
\dot{x}(t)=r x(t)\left(1-\frac{x(t)}{K}\right)-a x(t) S(t), \\
\dot{S}(t)=b x(t-\tau) S(t-\tau)-c S^{2}(t)-\frac{\beta S(t) I(t)}{1+\alpha I(t)}, \\
\dot{I}(t)=\frac{\beta S(t) I(t)}{1+\alpha I(t)}-d I(t),
\end{gathered}
$$

with initial conditions

$$
\begin{gathered}
x(\theta)=\phi_{1}(\theta), \quad S(\theta)=\phi_{2}(\theta), \quad I(\theta)=\phi_{3}(\theta), \\
\phi_{i}(\theta) \geq 0, \quad \theta \in[-\tau, 0], \quad \phi_{i}(0)>0 \quad(i=1,2,3),
\end{gathered}
$$

where $\left.\left(\phi_{1}(\theta), \phi_{2}(\theta), \phi_{3}(\theta)\right) \in C([-\tau, 0]), \mathbb{R}_{+}^{3}\right)$, the Banach space of continuous functions mapping the interval $[-\tau, 0]$ into $\mathbb{R}_{+0}^{3}$, here $\mathbb{R}_{+0}^{3}=\left\{\left(x_{1}, x_{2}, x_{3}\right): x_{i} \geq 0, i=1,2,3\right\}$. 
We make the following assumptions for our model (1.1).

(A1) The prey population grows logistically with intrinsic growth rate $r$ and environmental carrying capacity $K$.

(A2) There is a spread of disease in predators which are divided solely into susceptible and infectious population. $a$ is the capturing rate of susceptible predators, $b$ is the growth rate of susceptible predator due to predation of prey.

(A3) Susceptible predators become infected when they come in contact with infected predator, and this contact process is assumed to follow the saturation incidence rate $\beta S(t) I(t) /(1+\alpha I(t))$, with $\beta$ measuring the force of infection and $\alpha$ the inhibition effect.

(A4) $c>0$ models death rate due to overcrowding, and $\tau$ is the time required for the gestation of susceptible predator. $d$ is the death rate of infected predator. All the above-mentioned parameters are assumed to be positive.

The paper is organized as follows. In the next section, the positivity of solutions and the permanence of system are discussed. By analyzing the corresponding characteristic equations, we find conditions for local stability and bifurcation results in Section 3. In Section 4, sufficient conditions are derived for the global stability of the disease-free planar equilibrium and the positive equilibrium of the system. Numerical examples are carried out to illustrate the validity of the main results. The paper ends with a conclusion in the last section.

\section{Permanence}

To prove the permanence of system (1.1), we need the following lemma, which is a direct application of Theorem 4.9.1 in the study by Kuang [17].

Lemma 2.1. Consider the following equation:

$$
\dot{x}(t)=a x(t-\tau)-b x(t)-c x^{2}(t),
$$

where $a, b, c, \tau>0$ and $x(t)>0$ for all $t \in[-\tau, 0]$.

(1) If $a>b$, then $\limsup \sup _{t \rightarrow+\infty} x(t)=(a-b) / c$.

(2) If $a<b$, then $\limsup _{t \rightarrow+\infty} x(t)=0$.

Theorem 2.2. All the solutions of (1.1) with initial conditions (1.2) are all nonnegative.

Proof. Let $(x(t), S(t), I(t))$ be the solution of system (1.1) satisfying conditions (1.2). From the first and last equations of system (1.1), we have

$$
\begin{aligned}
& x(t)=x(0) e^{\int_{0}^{t}[r(1-x(\xi) / K)-a S(\xi)] d \xi}, \\
& I(t)=I(0) e^{\int_{0}^{t}[\beta S(\xi) /(1+\alpha I(\xi))-d] d \xi} .
\end{aligned}
$$

Hence, $x(t)$ and $I(t)$ are positive. 
We now claim that $S(t)>0$ for all $t>0$. Otherwise, there exists a $t_{1}>0$ such that $S\left(t_{1}\right)=0$ and $S(t)>0$ for all $t \in\left[0, t_{1}\right)$. Then $\dot{S}\left(t_{1}\right) \leq 0$. From the second equation of (1.1), we have

$$
\dot{S}\left(t_{1}\right)=b x\left(t_{1}-\tau\right) S\left(t_{1}-\tau\right)>0,
$$

which is a contradiction.

Theorem 2.3. All the solutions of (1.1) with initial conditions (1.2) are ultimately bounded.

Proof. From the first equation of (1.1), we have

$$
\dot{x}(t) \leq r x(t)\left(1-\frac{x(t)}{K}\right) .
$$

Hence, we get

$$
\limsup _{t \rightarrow+\infty} x(t) \leq K \doteq M_{1}
$$

From the second equation of system (1.1), for $t$ sufficiently large, we have

$$
\begin{aligned}
\dot{S}(t) & =b x(t-\tau) S(t-\tau)-c S^{2}(t)-\frac{\beta S(t) I(t)}{1+\alpha I(t)} \\
& \leq b K S(t-\tau)-c S^{2}(t) .
\end{aligned}
$$

Hence, by Lemma 2.1, one can get

$$
\limsup _{t \rightarrow+\infty} S(t) \leq \frac{b K}{c} \doteq M_{2}
$$

It follows from the third equation of (1.1) and the above inequality, that for $t$ sufficiently large, we have

$$
\begin{aligned}
\dot{I}(t) & =\frac{\beta S(t) I(t)}{1+\alpha I(t)}-d I(t) \\
& \leq \frac{\beta M_{2} I(t)}{1+\alpha I(t)}-d I(t) .
\end{aligned}
$$

Hence, one can see $\limsup \sup _{t \rightarrow+\infty} I(t) \leq(1 /(d \alpha))\left|\beta M_{2}-d\right| \doteq M_{3}$.

Now, we show that system (1.1) is permanent.

Theorem 2.4. Suppose that

$\left(H_{1}\right)$

$$
\beta m_{2}>d
$$

where $m_{2}$ is defined in (2.13), then system (1.1) is permanent. 
Proof. From the first equation of system (1.1), we have

$$
\dot{x}(t) \geq r x(t)\left(1-\frac{x(t)}{K}-\frac{a M_{2}}{r}\right) .
$$

It then follows that

$$
\liminf _{t \rightarrow+\infty} x(t) \geq K\left[1-\frac{a M_{2}}{r}\right] \doteq m_{1}
$$

Using the second equation of system (1.1), for $t$ sufficiently large, we have

$$
\dot{S}(t) \geq b m_{1} S(t-\tau)-c S^{2}(t)-\frac{\beta S(t) M_{3}}{1+\alpha M_{3}} .
$$

Hence, by Lemma 2.1 and $\left(H_{1}\right)$, one can derive that

$$
\liminf _{t \rightarrow+\infty} S(t) \geq \frac{1}{c}\left[b m_{1}-\frac{\beta M_{3}}{1+\alpha M_{3}}\right] \doteq m_{2} .
$$

From the third equation of system (1.1) and, above inequality, we have

$$
\dot{I}(t) \geq \frac{\beta m_{2} I(t)}{1+\alpha I(t)}-d I(t) .
$$

Since $\left(H_{1}\right)$ holds, then

$$
\liminf _{t \rightarrow+\infty} I(t) \geq \frac{1}{d \alpha}\left[\beta m_{2}-d\right] \doteq m_{3}
$$

Therefore, the above calculations and Theorem 2.2 imply that there exist $M_{i}, m_{i}(i=$ $1,2,3)$ such that

$$
\begin{aligned}
& 0<m_{1} \leq \liminf _{t \rightarrow+\infty} x(t) \leq \limsup _{t \rightarrow+\infty} x(t) \leq M_{1}, \\
& 0<m_{2} \leq \liminf _{t \rightarrow+\infty} S(t) \leq \limsup _{t \rightarrow+\infty} S(t) \leq M_{2}, \\
& 0<m_{3} \leq \liminf _{t \rightarrow+\infty} I(t) \leq \limsup _{t \rightarrow+\infty} I(t) \leq M_{3} .
\end{aligned}
$$

\section{Local Stability}

System (1.1) possesses the following equilibria.

(1) The trivial equilibrium $E_{0}(0,0,0)$.

(2) The axial equilibrium $E_{1}(K, 0,0)$.

(3) The disease-free planar equilibrium $E_{2}\left(x_{2}, S_{2}, 0\right)$, where

$$
x_{2}=\frac{K c r}{K a b+r c}, \quad S_{2}=\frac{K b r}{K a b+r c} .
$$


(4) The unique positive equilibrium $E_{3}\left(x_{3}, S_{3}, I_{3}\right)$ exists if $\beta S_{3}>d$, where

$$
\begin{gathered}
S_{3}=\frac{K b \alpha-\beta+\sqrt{(K b \alpha-\beta)^{2}+4 d \alpha(K a b / r+c)}}{2 \alpha(K a b / r+c)}, \\
I_{3}=\frac{\beta S_{3}-d}{d \alpha}, \quad x_{3}=K-\frac{K a S_{3}}{r} .
\end{gathered}
$$

In the following, we discuss the local stability of each equilibrium of system (1.1) by analyzing the corresponding characteristic equations, respectively.

\subsection{Stability of Equilibrium $E_{0}$}

The characteristic equation of system (1.1) at the trivial equilibrium $E_{0}$ is of the form

$$
\lambda(\lambda-r)(\lambda+d)=0 .
$$

It is easy to see that (3.3) always has a positive root $r$. Hence, $E_{0}$ is always unstable.

\subsection{Stability of Equilibrium $E_{1}$}

The characteristic equation of system (1.1) at the axial equilibrium $E_{1}$ is of the form

$$
(\lambda+K)\left(\lambda-b K e^{-\lambda \tau}\right)(\lambda+d)=0 .
$$

There are two characteristic roots $\lambda_{1}=-K, \lambda_{2}=-d$, and another characteristic root is given by the root of

$$
\lambda=b K e^{-\lambda \tau}
$$

It is clear that $\operatorname{Re} \lambda>0$. Hence, $E_{1}$ is always unstable.

\subsection{Stability of Equilibrium $E_{2}$}

Theorem 3.1. The disease-free planar equilibrium $E_{2}$ is locally asymptotically stable if $\beta S_{2}<d$, and the equilibrium $E_{2}$ is unstable if $\beta S_{2}>d$.

Proof. The characteristic equation of system (1.1) at the disease-free planar equilibrium $E_{2}$ is of the form

$$
\left(\lambda+\frac{r x_{2}}{K}\right)\left(\lambda+2 c S_{2}-c S_{2} e^{-\lambda \tau}\right)\left(\lambda+d-\beta S_{2}\right)=0
$$

Clearly, $\lambda_{1}=-r x_{2} / K$ is a negative eigenvalue. The second eigenvalue is given by the root of

$$
\lambda_{2}=c S_{2}\left(e^{-\lambda_{2} \tau}-2\right)
$$


Suppose that $\operatorname{Re} \lambda_{2} \geq 0$, then $\operatorname{Re} \lambda_{2}=c S_{2}\left(e^{-\operatorname{Re} \lambda_{2} \tau} \cos \left(\tau \operatorname{Im} \lambda_{2}\right)-2\right)<0$. It is a contradiction, so $\operatorname{Re} \lambda_{2}<0$. The last eigenvalue is $\lambda_{3}=\beta S_{2}-d$. The equilibrium $E_{2}$ is locally asymptotically stable if $\beta S_{2}<d$, and the equilibrium $E_{2}$ is unstable if $\beta S_{2}>d$.

\subsection{Stability of Equilibrium $E_{3}$}

The characteristic equation of system (1.1) at the positive equilibrium $E_{3}$ is of the form

$$
\lambda^{3}+A_{1} \lambda^{2}+A_{2} \lambda+A_{3}+e^{-\lambda \tau}\left(B_{1} \lambda^{2}+B_{2} \lambda+B_{3}\right)=0
$$

where

$$
\begin{aligned}
& A_{1}=\frac{r x_{3}}{K}+2 c S_{3}+\frac{\beta I_{3}}{1+\alpha I_{3}}+\frac{d \alpha I_{3}}{1+\alpha I_{3}} \\
& A_{2}=\left(\frac{r x_{3}}{K}+\frac{d \alpha I_{3}}{1+\alpha I_{3}}\right) \times\left(2 c S_{3}+\frac{\beta I_{3}}{1+\alpha I_{3}}\right)+\frac{d \alpha I_{3}}{1+\alpha I_{3}} \times \frac{r x_{3}}{K}+\frac{\beta I_{3}}{\left(1+\alpha I_{3}\right)^{2}} \\
& A_{3}=\frac{r x_{3}}{K} \times\left[\frac{d \beta I_{3}}{1+\alpha I_{3}}+\left(2 c S_{3}+\frac{\beta I_{3}}{1+\alpha I_{3}}\right) \times \frac{d \alpha I_{3}}{1+\alpha I_{3}}\right] \\
& B_{1}=-b x_{3}, \\
& B_{2}=b x_{3} \times\left(-\frac{d \alpha I_{3}}{1+\alpha I_{3}}-\frac{r x_{3}}{K}+a S_{3}\right) \\
& B_{3}=b x_{3} \times \frac{d \alpha I_{3}}{1+\alpha I_{3}} \times\left(-\frac{r x_{3}}{K}+a S_{3}\right) .
\end{aligned}
$$

For $\tau=0$, the transcendental (3.8) reduces to the following equation:

$$
\lambda^{3}+\left(A_{1}+B_{1}\right) \lambda^{2}+\left(A_{2}+B_{2}\right) \lambda+A_{3}+B_{3}=0 .
$$

We can easily get

$$
\begin{gathered}
A_{1}+B_{1}=\frac{r x_{3}}{K}+c S_{3}+\frac{d \alpha I_{3}}{1+\alpha I_{3}}>0, \\
A_{2}+B_{2}=\left(\frac{r x_{3}}{K}+\frac{d \alpha I_{3}}{1+\alpha I_{3}}\right) \times c S_{3}+\frac{d \alpha I_{3}}{1+\alpha I_{3}} \times \frac{r x_{3}}{K}+\frac{\beta I_{3}}{\left(1+\alpha I_{3}\right)^{2}}+b x_{3} a S_{3}>0, \\
A_{3}+B_{3}=\frac{r x_{3}}{K} \times\left[\frac{d \beta I_{3}}{1+\alpha I_{3}}+c S_{3} \times \frac{d \alpha I_{3}}{1+\alpha I_{3}}\right]+b x_{3} \times \frac{d \alpha I_{3}}{1+\alpha I_{3}} \times a S_{3}>0, \\
{\left[A_{1}+B_{1}\right] \times\left[A_{2}+B_{2}\right]-\left[A_{3}+B_{3}\right]>0 .}
\end{gathered}
$$

Therefore, the Routh-Hurwitz criterion implies that all the roots of (3.8) have negative real parts and we can conclude that the positive equilibrium $E_{3}$ is asymptotically stable in the absence of delay. 
Theorem 3.2. For system (1.1), if the condition $\left(H_{2}\right) A_{3}<B_{3}$ holds, the positive equilibrium $E_{3}$ is conditionally stable.

Proof. Substituting $\lambda=i \omega$ into (3.8) and separating the real and imaginary parts, one can get

$$
\begin{aligned}
& A_{1} \omega^{2}-A_{3}=\left(B_{3}-B_{1} \omega_{2}\right) \cos (\omega \tau)+B_{2} \omega \sin (\omega \tau), \\
& \omega^{3}-A_{2} \omega=B_{2} \omega \cos (\omega \tau)-\left(B_{3}-B_{1} \omega_{2}\right) \sin (\omega \tau) .
\end{aligned}
$$

Squaring and adding (3.12) we get

$$
\omega^{6}+D_{1} \omega^{4}+D_{2} \omega^{2}+D_{3}=0
$$

where

$$
D_{1}=A_{1}^{2}-2 A_{2}-B_{1}^{2}, \quad D_{2}=A_{2}^{2}-B_{2}^{2}-2 A_{1} A_{3}+2 B_{1} B_{3}, \quad D_{3}=A_{3}^{2}-B_{3}^{2} .
$$

We know that $D_{3}<0$ provided that the condition $\left(H_{2}\right)$ holds. There is at least a positive $\omega_{0}$ satisfying (3.13), that is, the characteristic equation (3.8) has a pair of purely imaginary roots of the form $\pm i \omega_{0}$. From (3.12), we can get the corresponding $\tau_{k}>0$ such that the characteristic (3.8) has a pair of purely imaginary roots

$$
\tau_{k}=\frac{1}{\omega_{0}} \arccos \left[\frac{\left(A_{1} \omega_{0}^{2}-A_{3}\right)\left(B_{3}-B_{1} \omega_{0}^{2}\right)+\left(\omega_{0}^{3}-A_{2} \omega_{0}\right) B_{2} \omega_{0}}{\left(B_{3}-B_{1} \omega_{0}^{2}\right)^{2}+\left(B_{2} \omega_{0}\right)^{2}}\right]+\frac{2 k \pi}{\omega_{0}}, \quad(k=0,1,2, \ldots) .
$$

Let $\lambda(\tau)=v(\tau)+i \omega(\tau)$ be the roots of (3.8) such that $\tau=\tau_{k}$ satisfying $v\left(\tau_{k}\right)=0$ and $\omega\left(\tau_{k}\right)=$ $\omega_{0}$. Differentiating the two sides of (3.8) with respect to $\tau$, we get

$$
\left(\frac{\mathrm{d} \lambda}{\mathrm{d} \tau}\right)^{-1}=\frac{2 \lambda^{3}+A_{1} \lambda^{2}-A_{2}}{-\lambda^{2}\left(\lambda^{3}+A_{1} \lambda^{2}+A_{2} \lambda+A_{3}\right)}+\frac{B_{1} \lambda^{2}-B_{3}}{\lambda^{2}\left(B_{1} \lambda^{2}+B_{2} \lambda+B_{3}\right)}-\frac{\tau}{\lambda}
$$

Therefore,

$$
\begin{aligned}
\operatorname{sign}\left[\frac{\mathrm{d} \operatorname{Re} \lambda}{\mathrm{d} \tau}\right]_{\tau=\tau_{k}} & =\operatorname{sign}\left[\operatorname{Re}\left(\frac{\mathrm{d} \lambda}{\mathrm{d} \tau}\right)^{-1}\right]_{\lambda=i \omega_{0}} \\
& =\frac{1}{\omega_{0}^{2}} \operatorname{sign}\left[\operatorname{Re}\left(\frac{A_{3}+A_{1} \omega_{0}^{2}+i 2 \omega_{0}^{3}}{A_{1} \omega_{0}^{2}-A_{3}+i\left(\omega_{0}^{3}-A_{2} \omega_{0}\right)}+\frac{B_{1} \omega_{0}^{2}+B_{3}}{-B_{1} \omega_{0}^{2}+i B_{2} \omega_{0}+B_{3}}\right)\right] \\
& =\frac{1}{\omega_{0}^{2}} \operatorname{sign}\left[\frac{2 \omega_{0}^{6}+\left(A_{1}^{2}-2 A_{2}-B_{1}^{2}\right) \omega_{0}^{4}+B_{3}^{2}-A_{3}^{2}}{\left(B_{3}-B_{1} \omega_{0}^{2}\right)^{2}+\left(B_{2} \omega_{0}\right)^{2}}\right] .
\end{aligned}
$$


If the conditions $\left(H_{2}\right)$ and $\left(H_{3}\right) A_{1}^{2}-2 A_{2}>B_{1}^{2}$ hold, one can see

$$
\operatorname{sign}\left[\frac{\mathrm{d} \operatorname{Re} \lambda}{\mathrm{d} \tau}\right]_{\tau=\tau_{k}}>0
$$

Therefore, the transversality condition holds, hence, the Hopf bifurcation occurs at $\omega=\omega_{0}$ and $\tau=\tau_{k}$.

Theorem 3.3. Suppose that the conditions $\left(H_{2}\right)$ and $\left(H_{3}\right)$ are satisfied.

(1) The positive equilibrium $E_{3}$ of system (1.1) is asymptotically stable for all $\tau \in\left[0, \tau_{0}\right)$ and unstable for $\tau>\tau_{0}$.

(2) System (1.1) undergoes a Hopf Bifurcation at the positive equilibrium $E_{3}$ when $\tau=\tau_{k}(k=$ $0,1, \ldots)$.

\section{Global Stability}

In this section, we study the global stability of equilibriums $E_{2}$ and $E_{3}$. The strategy of proofs is to use an iteration technique and comparison arguments, respectively.

\section{Theorem 4.1. If}

$\left(H_{4}\right) \beta b K<c d, K a b<r c$ holds, then the disease-free planar equilibrium $E_{2}$ is globally asymptotically stable.

Proof. Let $(x(t), S(t), I(t))$ be any positive solution of system (1.1) with initial conditions (1.2). Let the following hold:

$$
\begin{gathered}
U_{1}=\limsup _{t \rightarrow+\infty} x(t), \quad U_{2}=\limsup _{t \rightarrow+\infty} S(t), \quad U_{3}=\limsup _{t \rightarrow+\infty} I(t), \\
V_{1}=\liminf _{t \rightarrow+\infty} x(t), \quad V_{2}=\liminf _{t \rightarrow+\infty} S(t), \quad V_{3}=\liminf _{t \rightarrow+\infty} I(t) .
\end{gathered}
$$

In the following we shall claim that $U_{1}=V_{1}=x_{2}, U_{2}=V_{2}=S_{2}, U_{3}=V_{3}=0$.

It follows from the first equation of system (1.1) that

$$
\dot{x}(t) \leq r x(t)\left(1-\frac{x(t)}{K}\right) .
$$

By comparison, we obtain that

$$
U_{1}=\limsup _{t \rightarrow+\infty} x(t) \leq K+\varepsilon
$$

Since this inequality holds true for arbitrary $\varepsilon>0$ sufficiently small, we conclude that $U_{1} \leq$ $M_{1}^{x}$, where

$$
M_{1}^{x}=K
$$

Hence, for $\varepsilon>0$ sufficiently small, there is a $T_{1}>0$ such that, if $t>T_{1}, x(t) \leq M_{1}^{x}+\varepsilon$. 
We, therefore, derive from the second equation of system (1.1) that, for $t>T_{1}+\tau$,

$$
\dot{S}(t) \leq b\left(M_{1}^{x}+\varepsilon\right) S(t-\tau)-c S^{2}(t)
$$

Hence, by Lemma 2.1, one can get

$$
U_{2}=\limsup _{t \rightarrow+\infty} S(t) \leq \frac{b\left(M_{1}^{x}+\varepsilon\right)}{c} \doteq M_{1}^{S}
$$

Hence, for $\varepsilon>0$ sufficiently small, there is a $T_{2}>0$ such that, if $T_{2}>T_{1}+\tau, S(t) \leq M_{1}^{S}+\varepsilon$.

It follows from the third equation of system (1.1) that, for $t>T_{2}$,

$$
\dot{I}(t) \leq \frac{\beta\left(M_{1}^{S}+\varepsilon\right) I(t)}{1+\alpha I(t)}-d I(t) .
$$

Since $\left(H_{4}\right)$ holds, one can see

$$
U_{3}=\limsup _{t \rightarrow+\infty} I(t) \leq 0
$$

According to Theorem 2.2, we can get $\lim _{t \rightarrow+\infty} I(t)=U_{3}=V_{3}=0$.

We derive from the first equation of system (1.1) that, for $t>T_{2}+\tau$,

$$
\dot{x}(t) \geq r x(t)\left(1-\frac{x(t)}{K}-\frac{a\left(M_{1}^{S}+\varepsilon\right)}{r}\right) .
$$

By comparison we derive that

$$
V_{1}=\liminf _{t \rightarrow+\infty} x(t) \geq K\left[1-\frac{a\left(M_{1}^{S}+\varepsilon\right)}{r}\right] .
$$

Since this inequality holds true for arbitrary $\varepsilon>0$ sufficiently small, we conclude that $V_{1} \geq$ $N_{1}^{x}$, where

$$
N_{1}^{x}=K\left[1-\frac{a M_{1}^{S}}{r}\right]
$$

Hence, for $\varepsilon>0$ sufficiently small, there is a $T_{3}>0$ such that, if $T_{3}>T_{2}+\tau, x(t) \geq N_{1}^{x}-\varepsilon$.

We derive from the second equation of system (1.1) that, for $t>T_{3}$,

$$
\dot{S}(t) \geq b\left(N_{1}^{x}-\varepsilon\right) S(t-\tau)-c S^{2}(t)-\frac{\beta S(t) \varepsilon}{1+\alpha \varepsilon} .
$$

Hence, by Lemma 2.1, one can get 
Discrete Dynamics in Nature and Society

$$
V_{2}=\liminf _{t \rightarrow+\infty} S(t) \geq \frac{1}{c}\left[b\left(N_{1}^{x}-\varepsilon\right)-\frac{\beta \varepsilon}{1+\alpha \varepsilon}\right] .
$$

Since this is true for arbitrary $\varepsilon>0$ sufficiently small, we conclude that $V_{2} \geq N_{1}^{S}$, where

$$
N_{1}^{S}=\frac{b N_{1}^{x}}{c}
$$

Hence, for $\varepsilon>0$ sufficiently small, there is a $T_{4}>0$ such that, if $T_{4}>T_{3}+\tau, S(t) \geq N_{1}^{S}-\varepsilon$. Again, it follows from the first equation of system (1.2) that, for $t>T_{4}$,

$$
\dot{x}(t) \leq r x(t)\left(1-\frac{x(t)}{K}-\frac{a\left(N_{1}^{S}-\varepsilon\right)}{r}\right) .
$$

A comparison argument yields

$$
U_{1}=\limsup _{t \rightarrow+\infty} x(t) \leq K\left[1-\frac{a\left(N_{1}^{S}-\varepsilon\right)}{r}\right] .
$$

Since this inequality holds true for arbitrary $\varepsilon>0$ sufficiently small, we conclude that $U_{1} \leq$ $M_{2}^{x}$, where

$$
M_{2}^{x}=K\left[1-\frac{a N_{1}^{S}}{r}\right] \text {. }
$$

Hence, for $\varepsilon>0$ sufficiently small, there is a $T_{5}>0$ such that, if $T_{5}>T_{4}+\tau, x(t) \leq M_{2}^{x}+\varepsilon$.

It follows from the second equation of system (1.1) that, for $t>T_{5}$,

$$
\dot{S}(t) \leq b\left(M_{2}^{x}+\varepsilon\right) S(t-\tau)-c S^{2}(t)-\frac{\beta S(t) \varepsilon}{1+\alpha \varepsilon} .
$$

By Lemma 2.1, one can derive that

$$
V_{2}=\limsup _{t \rightarrow+\infty} S(t) \leq \frac{1}{c}\left[b\left(M_{2}^{x}+\varepsilon\right)-\frac{\beta \varepsilon}{1+\alpha \varepsilon}\right]
$$

Since this is true for arbitrary $\varepsilon>0$ sufficiently small, we conclude that $U_{2} \leq M_{2}^{S}$, where

$$
M_{2}^{S}=\frac{b M_{2}^{x}}{c} .
$$

Hence, for $\varepsilon>0$ sufficiently small, there is a $T_{6}>0$ such that, if $T_{6}>T_{5}+\tau, S(t) \leq M_{2}^{S}+\varepsilon$. We derive from the first equation of system (1.1) that, for $t>T_{6}$, 


$$
\dot{x}(t) \geq r x(t)\left(1-\frac{x(t)}{K}-\frac{a\left(M_{2}^{S}+\varepsilon\right)}{r}\right) .
$$

By comparison it follows that

$$
V_{1}=\liminf _{t \rightarrow+\infty} x(t) \geq K\left[1-\frac{a\left(M_{2}^{S}+\varepsilon\right)}{r}\right] .
$$

Since this inequality holds true for arbitrary $\varepsilon>0$ sufficiently small, we conclude that $V_{1} \geq$ $N_{2}^{x}$, where

$$
N_{2}^{x}=K\left[1-\frac{a M_{2}^{S}}{r}\right] .
$$

Hence, for $\varepsilon>0$ sufficiently small, there is a $T_{7}>0$ such that, if $T_{7}>T_{6}+\tau, x(t) \geq N_{2}^{x}-\varepsilon$.

We derive from the second equation of system (1.1) that, for $t>T_{7}$,

$$
\dot{S}(t) \geq b\left(N_{2}^{x}-\varepsilon\right) S(t-\tau)-c S^{2}(t)-\frac{\beta S(t) \varepsilon}{1+\alpha \varepsilon} .
$$

Hence, by Lemma 2.1, one can get

$$
V_{2}=\liminf _{t \rightarrow+\infty} S(t) \geq \frac{1}{c}\left[b\left(N_{2}^{x}-\varepsilon\right)-\frac{\beta \varepsilon}{1+\alpha \varepsilon}\right] .
$$

Since this inequality holds true for arbitrary $\varepsilon>0$ sufficiently small, we conclude that $V_{2} \geq$ $N_{2}^{S}$, where

$$
N_{2}^{S}=\frac{b N_{2}^{x}}{c}
$$

Hence, for $\varepsilon>0$ sufficiently small, there is a $T_{7}>0$ such that, if $T_{8}>T_{7}+\tau, S(t) \geq N_{2}^{S}-\varepsilon$.

Continuing this process, we get four sequences $M_{n}^{x}, M_{n}^{S}, N_{n}^{x}, N_{n}^{S}(n=1,2, \ldots)$ such that, for $n \geq 2$,

$$
\begin{aligned}
& M_{n}^{x}=K\left[1-\frac{a N_{n-1}^{S}}{r}\right], \\
& N_{n}^{x}=K\left[1-\frac{a M_{n}^{S}}{r}\right], \\
& M_{n}^{S}=\frac{b M_{n}^{x}}{c}, \\
& N_{n}^{S}=\frac{b N_{n}^{x}}{c} .
\end{aligned}
$$


Clearly, we have

$$
N_{n}^{x} \leq V_{1} \leq U_{1} \leq M_{n}^{x}, \quad N_{n}^{S} \leq V_{2} \leq U_{2} \leq M_{n}^{S} .
$$

It follows from (4.27) that

$$
M_{n+1}^{x}=K\left[1-\frac{K a b}{r c}\right]+M_{n}^{x} \frac{K^{2} a^{2} b^{2}}{r^{2} c^{2}} .
$$

Noting that $M_{n}^{x} \geq S_{2}$ and $K a b<r c$, we derive from (4.29) that

$$
\begin{aligned}
M_{n+1}^{x} & =K\left[1-\frac{K a b}{r c}\right]+M_{n}^{x}\left[\frac{K a b}{r c}-1\right]\left[\frac{K a b}{r c}+1\right] \\
& \leq K\left[1-\frac{K a b}{r c}\right]+\frac{K c r}{K a b+r c}\left[\frac{K a b}{r c}-1\right]\left[\frac{K a b}{r c}+1\right] \\
& =0 .
\end{aligned}
$$

Thus, the sequence $M_{n}^{x}$ is monotonically nonincreasing. Therefore, it follows that $\lim _{n \rightarrow+\infty} M_{n}^{x}$ exists. Taking $n \rightarrow+\infty$, we obtain from (4.29) that

$$
\lim _{n \rightarrow+\infty} M_{n+1}^{x}=K\left[1-\frac{K a b}{r c}\right]+\lim _{n \rightarrow+\infty} M_{n}^{x} \frac{K^{2} a^{2} b^{2}}{r^{2} c^{2}} .
$$

Noting that

$$
\lim _{n \rightarrow+\infty} M_{n+1}^{x}=\lim _{n \rightarrow+\infty} M_{n}^{x}
$$

it follows from (4.31) that

$$
\lim _{n \rightarrow+\infty} M_{n+1}^{x}=\lim _{n \rightarrow+\infty} M_{n}^{x}=x_{2}
$$

We derive from (4.33) and the third equation of (4.27) that

$$
\lim _{n \rightarrow+\infty} M_{n+1}^{S}=\lim _{n \rightarrow+\infty} M_{n}^{S}=S_{2}
$$

Similarly, one can derive from (4.27) and (4.34) that

$$
\lim _{n \rightarrow+\infty} N_{n}^{x}=x_{2}, \quad \lim _{n \rightarrow+\infty} N_{n}^{S}=S_{2} .
$$

It follows from (4.28), (4.33), and (4.35) that

$$
V_{1}=U_{1}=x_{2}, \quad V_{2}=U_{2}=S_{2} .
$$


We, therefore, have

$$
\lim _{t \rightarrow+\infty} x(t)=x_{2}, \quad \lim _{t \rightarrow+\infty} S(t)=S_{2}, \quad \lim _{t \rightarrow+\infty} I(t)=0 .
$$

Hence, the disease-free planar equilibrium $E_{2}$ is globally asymptotically stable. The proof is complete.

\section{Theorem 4.2. If}

$\left(H_{5}\right) \beta b K>c d$ and $K a b<r c, \beta>K b \alpha$ holds, then the positive equilibrium $E_{3}$ is globally asymptotically stable.

Proof. Let $(x(t), S(t), I(t))$ be any positive solution of system (1.1) with initial conditions (1.2). Let the following hold:

$$
\begin{aligned}
& \bar{x}=\limsup _{t \rightarrow+\infty} x(t), \quad \bar{S}=\limsup _{t \rightarrow+\infty} S(t), \quad \bar{I}=\limsup _{t \rightarrow+\infty} I(t), \\
& \underline{x}=\liminf _{t \rightarrow+\infty} x(t), \quad \underline{S}=\liminf _{t \rightarrow+\infty} S(t), \quad \underline{I}=\liminf _{t \rightarrow+\infty} I(t) .
\end{aligned}
$$

In the following we claim that $\bar{x}=\underline{x}=x_{3}, \bar{S}=\underline{S}=S_{3}, \bar{I}=\underline{I}=I_{3}$.

It follows from the first equation of system (1.1) that

$$
\dot{x}(t) \leq r x(t)\left(1-\frac{x(t)}{K}\right) .
$$

By comparison we obtain

$$
\bar{x}=\limsup _{t \rightarrow+\infty} x(t) \leq K+\varepsilon .
$$

Since this inequality holds true for arbitrary $\varepsilon>0$ sufficiently small, we conclude that $\bar{x} \leq M_{1}^{x}$, where

$$
M_{1}^{x}=K
$$

Hence, for $\varepsilon>0$ sufficiently small, there is a $T_{1}>0$ such that, if $t>T_{1}, x(t) \leq M_{1}^{x}+\varepsilon$. We obtain from the second equation of system (1.1) that, for $t>T_{1}+\tau$,

$$
\dot{S}(t) \leq b\left(M_{1}^{x}+\varepsilon\right) S(t-\tau)-c S^{2}(t) .
$$

Hence, by Lemma 2.1, we derive that

$$
\bar{S}=\limsup _{t \rightarrow+\infty} S(t) \leq \frac{b\left(M_{1}^{x}+\varepsilon\right)}{c} .
$$


Since it is true for arbitrary $\varepsilon>0$ sufficiently small, we conclude that $\bar{S} \leq M_{1}^{S}$, where

$$
M_{1}^{S}=\frac{b M_{1}^{x}}{c} .
$$

Hence, for $\varepsilon>0$ sufficiently small, there is a $T_{2}>0$ such that, if $T_{2}>T_{1}+\tau, S(t) \leq M_{1}^{S}+\varepsilon$.

It follows from the third equation of system (1.1) that

$$
\dot{I}(t) \leq \frac{\beta\left(M_{1}^{S}+\varepsilon\right) I(t)}{1+\alpha I(t)}-d I(t) .
$$

Since $\left(H_{5}\right)$ holds, one can see

$$
\bar{I}=\limsup _{t \rightarrow+\infty} I(t) \leq \frac{\beta\left(M_{1}^{S}+\varepsilon\right)-d}{d \alpha} .
$$

Since it is true for arbitrary $\varepsilon>0$ sufficiently small, we conclude that $\bar{I} \leq M_{1}^{I}$, where

$$
M_{1}^{I}=\frac{\beta M_{1}^{S}-d}{d \alpha} .
$$

We derive from the first equation of system (1.1) that, for $t>T_{2}$,

$$
\dot{x}(t) \geq r x(t)\left(1-\frac{x(t)}{K}-\frac{a\left(M_{1}^{S}+\varepsilon\right)}{r}\right) .
$$

By comparison we derive that

$$
\underline{x}=\liminf _{t \rightarrow+\infty} x(t) \geq K\left[1-\frac{a\left(M_{1}^{S}+\varepsilon\right)}{r}\right] .
$$

Since this inequality holds true for arbitrary $\varepsilon>0$ sufficiently small, we conclude that $\underline{x} \geq N_{1}^{x}$, where

$$
N_{1}^{x}=K\left[1-\frac{a M_{1}^{S}}{r}\right]
$$

Hence, for $\varepsilon>0$ sufficiently small, there is a $T_{3}>0$ such that, if $T_{3}>T_{2}+\tau, x(t) \geq N_{1}^{x}-\varepsilon$. We derive from the second equation of system (1.1) that, for $t>T_{3}$,

$$
\dot{S}(t) \geq b\left(N_{1}^{x}-\varepsilon\right) S(t-\tau)-c S^{2}(t)-\frac{\beta S(t) M_{1}^{I}}{1+\alpha M_{1}^{I}}
$$


Hence, by Lemma 2.1 and $\left(H_{5}\right)$, one can get

$$
\underline{S}=\liminf _{t \rightarrow+\infty} S(t) \geq \frac{1}{c}\left[b\left(N_{1}^{x}-\varepsilon\right)-\frac{\beta M_{1}^{I}}{1+\alpha M_{1}^{I}}\right] .
$$

Since this inequality holds true for arbitrary $\varepsilon>0$ sufficiently small, we conclude that $\underline{S} \geq N_{1}^{S}$, where

$$
N_{1}^{S}=\frac{1}{c}\left[b N_{1}^{x}-\frac{\beta M_{1}^{I}}{1+\alpha M_{1}^{I}}\right] .
$$

Hence, for $\varepsilon>0$ sufficiently small, we get $S(t) \geq N_{1}^{S}-\varepsilon$.

It follows from the third equation of system (1.1) that

$$
\dot{I}(t) \geq \frac{\beta\left(N_{1}^{S}-\varepsilon\right) I(t)}{1+\alpha I(t)}-d I(t) .
$$

Provided that $\beta N_{1}^{S}>d$, one can see

$$
\underline{I}=\liminf _{t \rightarrow+\infty} I(t) \geq \frac{\beta\left(N_{1}^{S}-\varepsilon\right)-d}{d \alpha} .
$$

Since this inequality holds true for arbitrary $\varepsilon>0$ sufficiently small, we conclude that $\underline{I} \geq N_{1}^{I}$, where

$$
N_{1}^{I}=\frac{\beta N_{1}^{S}-d}{d \alpha}
$$

It follows from the first equation of system (1.1) that

$$
\dot{x}(t) \leq r x(t)\left(1-\frac{x(t)}{K}-\frac{a\left(N_{1}^{S}-\varepsilon\right)}{r}\right) .
$$

By comparison we derive that

$$
\bar{x}=\limsup _{t \rightarrow+\infty} x(t) \leq K\left[1-\frac{a\left(N_{1}^{S}-\varepsilon\right)}{r}\right] .
$$

Since this inequality holds true for arbitrary $\varepsilon>0$ sufficiently small, we conclude that $\bar{x} \leq M_{2}^{x}$, where

$$
M_{2}^{x}=K\left[1-\frac{a N_{1}^{S}}{r}\right]
$$

Hence, for $\varepsilon>0$ sufficiently small, there is a $T_{4}>0$ such that, if $t>T_{4}, x(t) \leq M_{2}^{x}+\varepsilon$. 
Discrete Dynamics in Nature and Society

We obtain from the second equation of system (1.1) that, for $t>T_{4}+\tau$,

$$
\dot{S}(t) \leq b\left(M_{2}^{x}+\varepsilon\right) S(t-\tau)-c S^{2}(t)-\frac{\beta S(t) N_{1}^{I}}{1+\alpha N_{1}^{I}}
$$

Hence, by Lemma 2.1, one can get

$$
\bar{S}=\limsup _{t \rightarrow+\infty} S(t) \leq \frac{1}{c}\left[b\left(M_{2}^{x}+\varepsilon\right)-\frac{\beta N_{1}^{I}}{1+\alpha N_{1}^{I}}\right] .
$$

Since this inequality holds true for arbitrary $\varepsilon>0$ sufficiently small, we conclude that $\bar{S} \leq M_{2}^{S}$, where

$$
M_{2}^{S}=\frac{1}{c}\left[b M_{2}^{x}-\frac{\beta N_{1}^{I}}{1+\alpha N_{1}^{I}}\right]
$$

Hence, for $\varepsilon>0$ sufficiently small, there is a $T_{5}>0$ such that, if $T_{5}>T_{4}+\tau, S(t) \leq M_{2}^{S}+\varepsilon$.

It follows from the third equation of system (1.1) that

$$
\dot{I}(t) \leq \frac{\beta\left(M_{2}^{S}+\varepsilon\right) I(t)}{1+\alpha I(t)}-d I(t) .
$$

Hence, by $\left(H_{5}\right)$, one can see

$$
\bar{I}=\limsup _{t \rightarrow+\infty} I(t) \leq \frac{\beta\left(M_{2}^{S}+\varepsilon\right)-d}{d \alpha} .
$$

Since this inequality holds true for arbitrary $\varepsilon>0$ sufficiently small, we conclude that $\bar{I} \leq M_{2}^{I}$, where

$$
M_{2}^{I}=\frac{\beta M_{2}^{S}-d}{d \alpha}
$$

We derive from the first equation of system (1.1) that, for $t>T_{5}$,

$$
\dot{x}(t) \geq r x(t)\left(1-\frac{x(t)}{K}-\frac{a\left(M_{2}^{S}+\varepsilon\right)}{r}\right) .
$$

By comparison we derive that

$$
\underline{x}=\liminf _{t \rightarrow+\infty} x(t) \geq K\left[1-\frac{a\left(M_{2}^{S}+\varepsilon\right)}{r}\right] .
$$


Since this inequality holds true for arbitrary $\varepsilon>0$ sufficiently small, we conclude that $\underline{x} \geq N_{2}^{x}$, where

$$
N_{2}^{x}=K\left[1-\frac{a M_{2}^{S}}{r}\right]
$$

Hence, for $\varepsilon>0$ sufficiently small, there is a $T_{6}>0$ such that, if $T_{6}>T_{5}+\tau, x(t) \geq N_{2}^{x}-\varepsilon$.

We derive from the second equation of system (1.1) that, for $t>T_{6}$,

$$
\dot{S}(t) \geq b\left(N_{2}^{x}-\varepsilon\right) S(t-\tau)-c S^{2}(t)-\frac{\beta S(t) M_{2}^{I}}{1+\alpha M_{2}^{I}}
$$

By Lemma 2.1, one can get

$$
\underline{S}=\liminf _{t \rightarrow+\infty} S(t) \geq \frac{1}{c}\left[b\left(N_{2}^{x}-\varepsilon\right)-\frac{\beta M_{2}^{I}}{1+\alpha M_{2}^{I}}\right] .
$$

Since this inequality holds true for arbitrary $\varepsilon>0$ sufficiently small, we conclude that $\underline{S} \geq N_{1}^{S}$, where

$$
N_{2}^{S}=\frac{1}{c}\left[b N_{2}^{x}-\frac{\beta M_{2}^{I}}{1+\alpha M_{2}^{I}}\right]
$$

Hence, for $\varepsilon>0$ sufficiently small, we get $S(t) \geq N_{2}^{S}-\varepsilon$.

It follows from the third equation of system (1.1) that

$$
\dot{I}(t) \geq \frac{\beta\left(N_{2}^{S}-\varepsilon\right) I(t)}{1+\alpha I(t)}-d I(t)
$$

Since $\left(H_{5}\right)$ holds, one can see

$$
\underline{I}=\liminf _{t \rightarrow+\infty} I(t) \geq \frac{\beta\left(N_{2}^{S}-\varepsilon\right)-d}{d \alpha} .
$$

Since this inequality holds true for arbitrary $\varepsilon>0$ sufficiently small, we conclude that $\underline{I} \geq N_{2}^{I}$, where

$$
N_{2}^{I}=\frac{\beta N_{2}^{S}-d}{d \alpha}
$$


Continuing this process, we obtain six sequences $M_{n}^{x}, M_{n}^{S}, M_{n}^{I}, N_{n}^{x}, N_{n}^{S}, N_{n}^{I}(n=$ $1,2, \ldots)$ such that, for $n \geq 2$,

$$
\begin{aligned}
& M_{n}^{x}=K\left[1-\frac{a N_{n-1}^{S}}{r}\right], \\
& M_{n}^{S}=\frac{1}{c}\left[b M_{n}^{x}-\frac{\beta N_{n-1}^{I}}{1+\alpha N_{n-1}^{I}}\right], \\
& M_{n}^{I}=\frac{\beta M_{n}^{S}-d}{d \alpha}, \\
& N_{n}^{x}=K\left[1-\frac{a M_{n}^{S}}{r}\right], \\
& N_{n}^{S}=\frac{1}{c}\left[b N_{n}^{x}-\frac{\beta M_{n}^{I}}{1+\alpha M_{n}^{I}}\right], \\
& N_{n}^{I}=\frac{\beta N_{n}^{S}-d}{d \alpha} .
\end{aligned}
$$

A direct calculation shows that

$$
\begin{aligned}
& M_{2}^{x}-M_{1}^{x}=-\frac{K a N_{1}^{S}}{r}<0, \\
& M_{2}^{S}-M_{1}^{S}=\frac{1}{c}\left[b\left(M_{2}^{x}-M_{1}^{x}\right)-\frac{\beta N_{1}^{I}}{1+\alpha N_{1}^{I}}\right]<0 \\
& M_{2}^{I}-M_{1}^{I}=\frac{\beta\left(M_{2}^{S}-M_{1}^{S}\right)}{d \alpha}<0 \\
& N_{2}^{x}-N_{1}^{x}=\frac{K a\left(M_{1}^{S}-M_{2}^{S}\right)}{r}>0, \\
& N_{2}^{S}-N_{1}^{S}=\frac{1}{c}\left[b\left(N_{2}^{x}-N_{1}^{x}\right)+\frac{\beta M_{1}^{I}}{1+\alpha M_{1}^{I}}-\frac{\beta M_{2}^{I}}{1+\alpha M_{2}^{I}}\right]>0, \\
& N_{2}^{I}-N_{1}^{I}=\frac{\beta\left(N_{2}^{S}-N_{1}^{S}\right)}{d \alpha}>0 .
\end{aligned}
$$

By induction, we can show that $M_{n+1}^{S}<M_{n}^{S}, N_{n+1}^{S}>N_{n}^{S}$. Therefore, the sequence $M_{n}^{S}$ is decreasing, and the sequence $N_{n}^{S}$ is increasing. Clearly, we have

$$
N_{n}^{x} \leq \underline{x} \leq \bar{x} \leq M_{n}^{x}, \quad N_{n}^{S} \leq \underline{S} \leq \bar{S} \leq M_{n}^{S}, \quad N_{n}^{I} \leq \underline{I} \leq \bar{I} \leq M_{n}^{I} .
$$

Hence, the limits of the sequences $M_{n}^{S}$ and $N_{n}^{S}$ exist. Denote

$$
\bar{S}=\lim _{t \rightarrow+\infty} M_{n}^{S}, \quad \underline{S}=\lim _{t \rightarrow+\infty} N_{n}^{S} .
$$




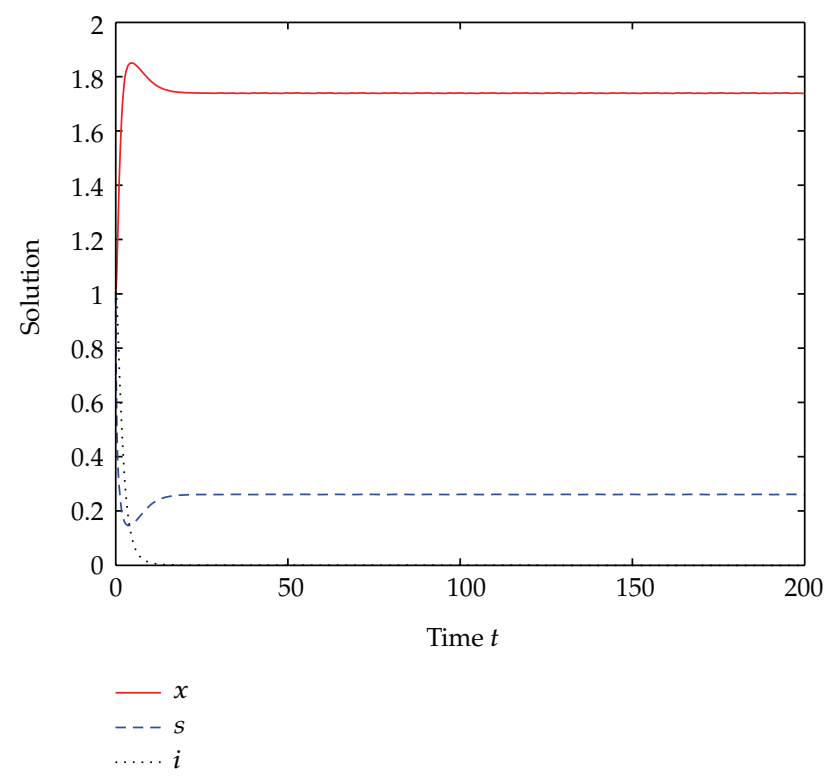

Figure 1: The temporal solution found by numerical integration of system (1.1) with $r=2, K=2, a=2$, $c=2, \beta=2, \alpha=1, d=0.8, b=0.3, \tau=1$, and $\left(x_{0}, S_{0}, I_{0}\right)=(1,1,1)$.

We derive from (4.75) that

$$
[\bar{S}-\underline{S}]\left[\beta-K b \alpha+\frac{K b a}{d r}(\bar{S}+\underline{S})\right]=0 .
$$

Since $\left(H_{5}\right)$ holds, $\beta-K b \alpha+(K b a / d r)(\bar{S}+\underline{S})>0$. It, therefore, follows from (4.79) that $\bar{S}=\underline{S}$. Accordingly, we derive from (4.75) that

$$
\bar{S}=\frac{1}{c}\left[b K\left(1-\frac{a \bar{S}}{r}\right)-\frac{\beta}{\alpha}+\frac{d}{\bar{S}}\right]
$$

By a simple calculation, we obtain

$$
\bar{S}=\underline{S}=S_{3} .
$$

It follows from (4.75) and (4.81) that $\bar{I}=\underline{I}=I_{3}, \bar{x}=\underline{x}=x_{3}$. Hence, the unique positive equilibrium $E_{3}$ is globally asymptotically stable. The proof is complete.

In the following we will present two examples to verify our results obtained earlier.

Example 4.3. In system (1.1), we let $r=2, K=2, a=1, c=2, \beta=2, \alpha=1, d=0.8, b=0.3$, $\tau=1$. It is easy to show that $K b \beta-c d=-0.4<0, r c-K a b=3.4>0$. By Theorem 4.1 we see that the equilibrium $E_{2}(1.7391,0.2609,0)$ of system (1.1) is globally stable (see Figure 1). 


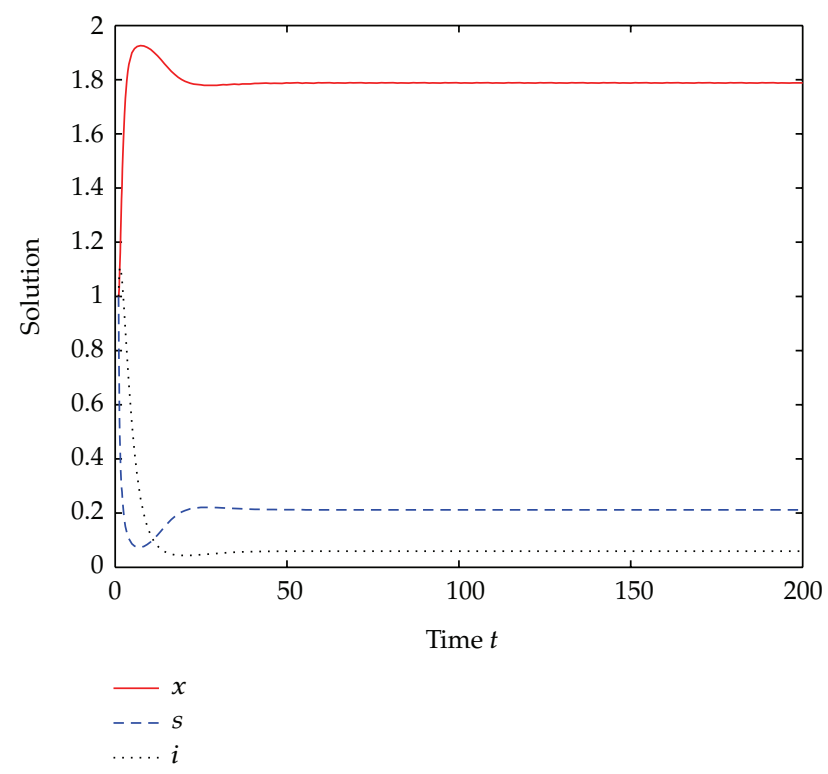

Figure 2: The temporal solution found by numerical integration of system (1.1) with $r=2, K=2, a=1$, $c=2, \beta=2, \alpha=1, d=0.4, b=0.3, \tau=1$, and $\left(x_{0}, S_{0}, I_{0}\right)=(1,1,1)$.

Example 4.4. In system (1.1), we let $r=2, K=2, a=1, c=2, \beta=2, \alpha=1, d=0.4, b=0.3, \tau=1$. It is easy to show that $K b \beta-c d=0.4>0, K a b-r c=-3.4<0, \beta-K b \alpha=1.400>0$. By Theorem 4.2 we see that the equilibrium $E_{3}(1.7881,0.2119,0.0596)$ of system (1.1) is globally stable, as depicted in Figure 2.

\section{Conclusion}

In this paper, we have incorporated the disease for the predator and the time delay into an eco-epidemiology model. A saturation incidence function was used to model the behavioral change of the susceptible predator when their number increases or due to the crowding effect of the infected predator. First, by comparison arguments, the permanence of system (1.1) was studied. Then, by analyzing the corresponding characteristic equations, sufficient conditions were derived for the local stability of each equilibrium of system (1.1). From Theorem 3.3, we showed that system (1.1) undergoes a Hopf bifurcation when the delay passes through a sequence of critical values. Next, by using the iteration technique and comparison arguments, we derived sufficient conditions for the global stability of the disease-free planer equilibrium and positive equilibrium of system (1.1). By Theorems 4.1 and 4.2, we showed that (1) if $\left(H_{4}\right)$ holds, the infected predator population becomes extinct and the disease will be eliminated; that is, only sound predator and prey coexist; $(2)$ if $\left(H_{5}\right)$ holds, the prey, the sound predator and the infected predator coexist. The disease will not be eliminated, and the system is permanent.

\section{Acknowledgment}

This work was supported by the National Natural Science Foundation of China (no. 11071254). 


\section{References}

[1] K. Kundu and J. Chattopadhyay, "A ratio-dependent eco-epidemiological model of the Salton Sea," Mathematical Methods in the Applied Sciences, vol. 29, no. 2, pp. 191-207, 2006.

[2] K. P. Das, S. Roy, and J. Chattopadhyay, "Effect of disease-selective predation on prey infected by contact and external sources," BioSystems, vol. 95, no. 3, pp. 188-199, 2009.

[3] X. Zhou and J. Cui, "Stability and Hopf bifurcation analysis of an eco-epidemiological model with delay," Journal of the Franklin Institute, vol. 347, no. 9, pp. 1654-1680, 2010.

[4] B. Mukhopadhyay and R. Bhattacharyya, "Role of predator switching in an eco-epidemiological model with disease in the prey," Ecological Modelling, vol. 220, no. 7, pp. 931-939, 2009.

[5] X. Zhou, X. Shi, and X. Song, "The dynamics of an eco-epidemiological model with distributed delay," Nonlinear Analysis: Hybrid Systems, vol. 3, no. 4, pp. 685-699, 2009.

[6] N. Bairagi, R. R. Sarkar, and J. Chattopadhyay, "Impacts of incubation delay on the dynamics of an eco-epidemiological system-a theoretical study," Bulletin of Mathematical Biology, vol. 70, no. 7, pp. 2017-2038, 2008.

[7] D. Mukherjee, "Hopf bifurcation in an eco-epidemic model," Applied Mathematics and Computation, vol. 217, no. 5, pp. 2118-2124, 2010.

[8] X. Zhou, X. Shi, and X. Song, "Analysis of a delay prey-predator model with disease in the prey species only," Journal of the Korean Mathematical Society, vol. 46, no. 4, pp. 713-731, 2009.

[9] J.-F. Zhang, W.-T. Li, and X.-P. Yan, "Hopf bifurcation and stability of periodic solutions in a delayed eco-epidemiological system," Applied Mathematics and Computation, vol. 198, no. 2, pp. 865-876, 2008.

[10] M. Haque and D. Greenhalgh, "A predator-prey model with disease in the prey species only," Mathematical Methods in the Applied Sciences, vol. 30, no. 8, pp. 911-929, 2007.

[11] L. Han, Z. Ma, and H. W. Hethcote, "Four predator prey models with infectious diseases," Mathematical and Computer Modelling, vol. 34, no. 7-8, pp. 849-858, 2001.

[12] H. W. Hethcote, W. Wang, L. Han, and Z. Ma, "A predator-prey model with infected prey," Theoretical Population Biology, vol. 66, no. 3, pp. 259-268, 2004.

[13] V. Capasso and G. Serio, "A generalization of the Kermack-McKendrick deterministic epidemic model," Mathematical Biosciences, vol. 42, no. 1-2, pp. 43-61, 1978.

[14] W. M. Liu, S. A. Levin, and Y. Iwasa, "Influence of nonlinear incidence rates upon the behavior of SIRS epidemiological models," Journal of Mathematical Biology, vol. 23, no. 2, pp. 187-204, 1986.

[15] S. Ruan and W. Wang, "Dynamical behavior of an epidemic model with a nonlinear incidence rate," Journal of Differential Equations, vol. 188, no. 1, pp. 135-163, 2003.

[16] R. Bhattacharyya and B. Mukhopadhyay, "On an eco-epidemiological model with prey harvesting and predator switching: local and global perspectives," Nonlinear Analysis: Real World Applications, vol. 11, no. 5, pp. 3824-3833, 2010.

[17] Y. Kuang, Delay Differential Equations with Applications in Population Dynamics, vol. 191 of Mathematics in Science and Engineering, Academic Press, London, UK, 1993. 


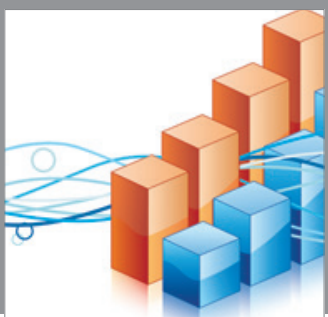

Advances in

Operations Research

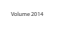

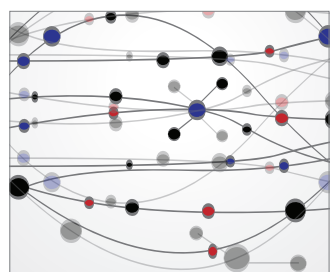

\section{The Scientific} World Journal
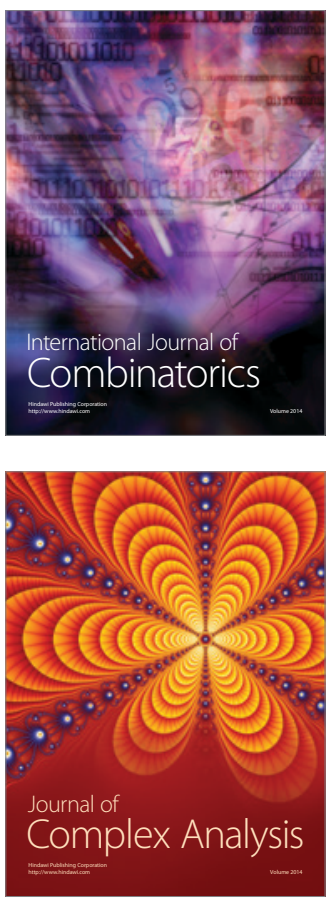

International Journal of

Mathematics and

Mathematical

Sciences
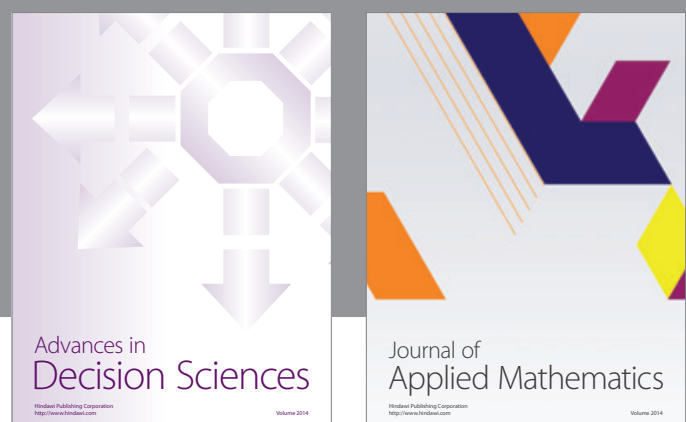

Journal of

Applied Mathematics
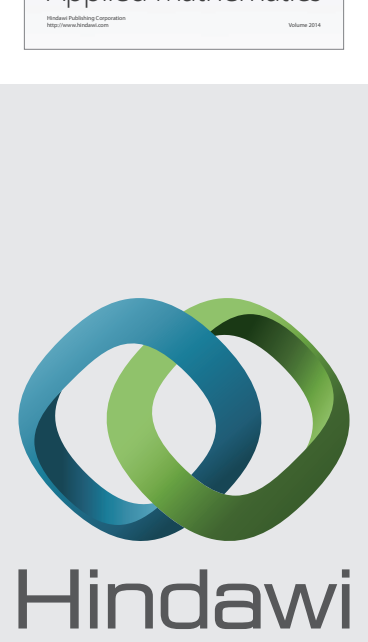

Submit your manuscripts at http://www.hindawi.com
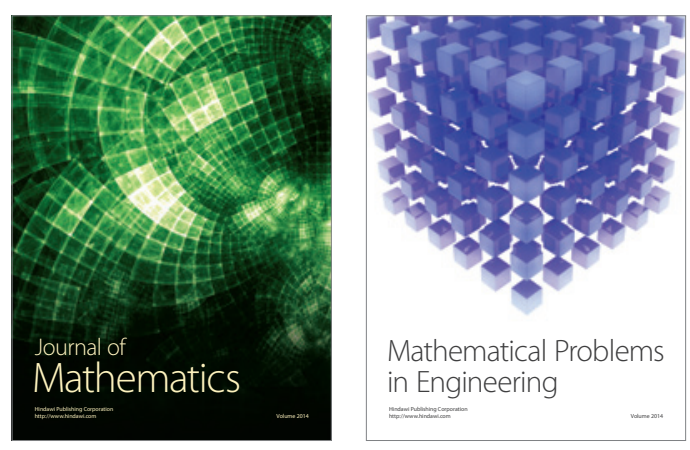

Mathematical Problems in Engineering
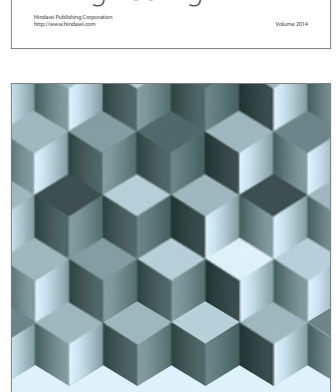

Journal of

Function Spaces
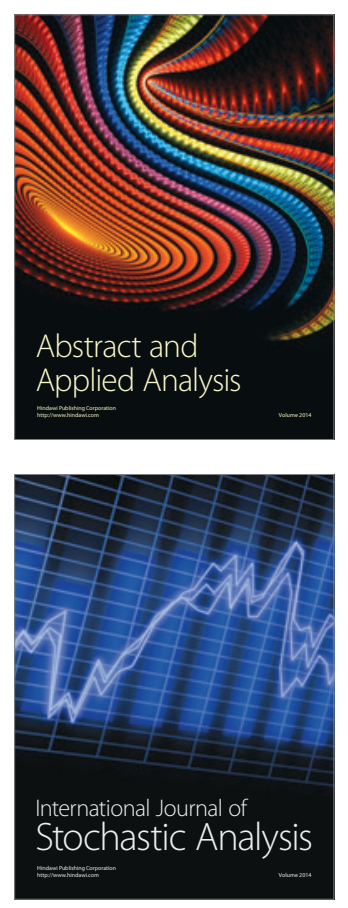

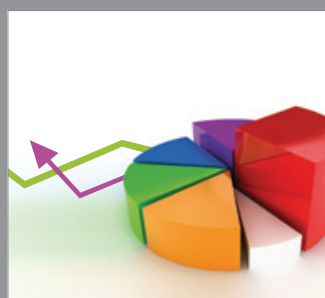

ournal of

Probability and Statistics

Promensencen
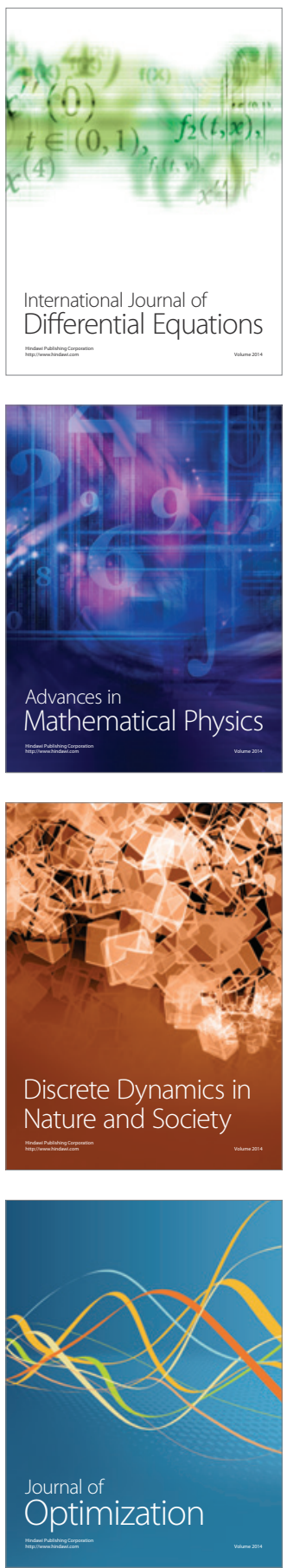